\title{
Structure characterization and strain relief analysis in CVD growth of boron phosphide on silicon carbide
}

Guoliang Li ${ }^{\text {a }}$, Julia K.C. Abbott ${ }^{\text {a }}$, John D. Brasfield ${ }^{\text {a }}$, Peizhi Liu ${ }^{\text {b }}$, Alexis Dale ${ }^{\text {a }}$, Gerd Duscher b, c, Philip D. Rack ${ }^{\text {b, d }}$, and Charles S. Feigerle ${ }^{a, *}$

${ }^{a}$ Department of Chemistry, The University of Tennessee, Knoxville, TN 37996, USA

${ }^{b}$ Department of Material Science and Engineering, The University of Tennessee, Knoxville, TN 37996, USA

${ }^{c}$ Materials Science and Technology Division, Oak Ridge National Laboratory, Oak Ridge, TN 37831, USA

${ }^{\mathrm{d}}$ Center for Nanophase Materials Sciences, Oak Ridge National Laboratory, Oak Ridge, TN 37831, USA

\begin{abstract}
Boron phosphide (BP) is a material of interest for development of a high-efficiency solid-state thermal neutron detector. For a thick film-based device, microstructure evolution is key to the engineering of material synthesis. Here, we report epitaxial BP films grown on silicon carbide with vicinal steps and provide a detailed analysis of the microstructure evolution and strain relief. The BP film is epitaxial in the near-interface region but deviates from epitaxial growth as the film develops. Defects such as coherent and incoherent twin boundaries, dislocation loops, stacking faults concentrate in the near-interface region and segment this region into small domains. The formation of defects in this region do not fully release the strain originated from
\end{abstract}


the lattice mismatch. Large grains emerge above the near-interface region and grain boundaries become the main defects in the upper part of the BP film.

\section{Keywords}

Boron phosphide, Defect, Strain relief, CVD, Neutron detection

* Corresponding author at: Department of Chemistry, The University of Tennessee, Knoxville, TN 37996-1600, USA.

Tel: +001 (865) 974-3141; fax: 865-974-9332.

Email: cfeigerl@tennessee.edu.

\section{Introduction}

Boron phosphide (BP) is an indirect bandgap semiconductor with potential for use as a solid state thermal neutron detector. ${ }^{10} \mathrm{~B}(20 \%$ of natural abundance boron) has a large neutron absorption cross section (3840 barns) and generates $2.3 \mathrm{MeV}$ of energy by the reaction [1],

$$
{ }^{10} \mathrm{~B}+n \rightarrow{ }^{7} \mathrm{Li}+\alpha+2.3 \mathrm{MeV} .
$$

To optimize the detection efficiency of a BP based neutron detector, it is important that BP be of high crystal quality and detrimental defects in the material be limited and not serve as charge traps or electron hole recombination sites. 
Most of previous work on chemical vapor deposition of BP was done on silicon (Si) [2-6] and sapphire substrates [7, 8]. Unfortunately, the poor lattice mismatches between $\mathrm{Si} / \mathrm{BP}(16.5 \%)$ and sapphire/BP (4.6\% and $11.5 \%$ depending on direction), are known to produce discontinuities across the interface and large amount of dislocations in the hetero-epilayer [8]. As an alternative, $\mathrm{SiC}$ has a $\{0001\}$ basal plane which offers only $4.5 \%$ mismatch with a BP $\{111\}$ plane and therefore should be a better substrate for BP epitaxy. In 1971, Chu et al. performed the first BP growth on $\mathrm{SiC}$, but characterized only the surface crystallinity using reflection electron diffraction [9]. In 2005, Udagawa et al. characterized a thin $<111>$ BP layer (200 440nm) grown on (0001) 6H-SiC using high-resolution transmission electron microscopy (HRTEM) [10]. They observed coherent twin boundaries and random atomic configurations at the $\mathrm{BP} / \mathrm{SiC}$ interface, but yet were unable to study the microstructure evolution in a larger scale due to the limited film thickness. In this paper, we present a detailed analysis of the defect generation, structural evolution and strain management in a thick BP film $(\sim 3 \mu \mathrm{m})$ grown on $4^{\circ}$ offcut $(0001) 4 \mathrm{H}-\mathrm{SiC}$.

\section{Experimental}

Heteroepitaxial BP films were grown by CVD on the (0001) surface of 4H-SiC substrates with a $4^{\mathrm{o}}$ offcut in a $<11-20>$ direction using diborane $\left(\mathrm{B}_{2} \mathrm{H}_{6}\right)$ and phosphine $\left(\mathrm{PH}_{3}\right)$ precursors. The reactor pressure was maintained at 630 Torr and the flow rates used were $20 \mathrm{sccm} \mathrm{B}_{2} \mathrm{H}_{6}(1 \%$ in $\left.\mathrm{H}_{2}\right), 100 \mathrm{sccm} \mathrm{PH}_{3}\left(5 \%\right.$ in $\left.\mathrm{H}_{2}\right)$ and $2500 \mathrm{sccm}$ of $\mathrm{H}_{2}$. Crystalline BP films have been grown at temperatures ranging from 800 to $950{ }^{\circ} \mathrm{C}$. The detailed structural and strain analysis presented here was on a film grown at $850{ }^{\circ} \mathrm{C}$. Details of the reactor design will be reported elsewhere. Angular off cut wafers were used to promote step-flow growth. 
TEM samples were prepared with a dual beam SEM/FIB Zeiss Auriga. High resolution phase contrast and Z-contrast imaging was performed using a Zeiss Libra $200 \mathrm{MC}$ transmission electron microscope and a VG HB 603 UX scanning transmission electron microscope. Both of the microscopes can reach Angstrom level resolution and reveal easily the $\{112\}$ and $\{111\}$ atomic planes in BP, which enables us to determine the microstructure. Strain and defects were analyzed utilizing electron micrographs, diffractograms and X-ray diffraction.

\section{Results and discussions}

\subsection{Structure overview of the BP film}

Fig. 1 is an overview bright-field image of the $\mathrm{BP} / \mathrm{SiC}$ cross-section taken under a $\mathrm{SiC}<11-20>$ zone-axis. The bright-field detector in TEM collects mostly the transmitted electrons, therefore, the more severe the electron diffraction the darker the image will be. Electron diffraction is particularly strong when a crystal is illuminated in zone-axis. As we can see in the image, the near-interface region has more dark contrast, while the upper part of the film has more bright contrast. This means BP grew epitaxially from the interface but deviated from epitaxial growth and became non-epitaxial as the film develops. 


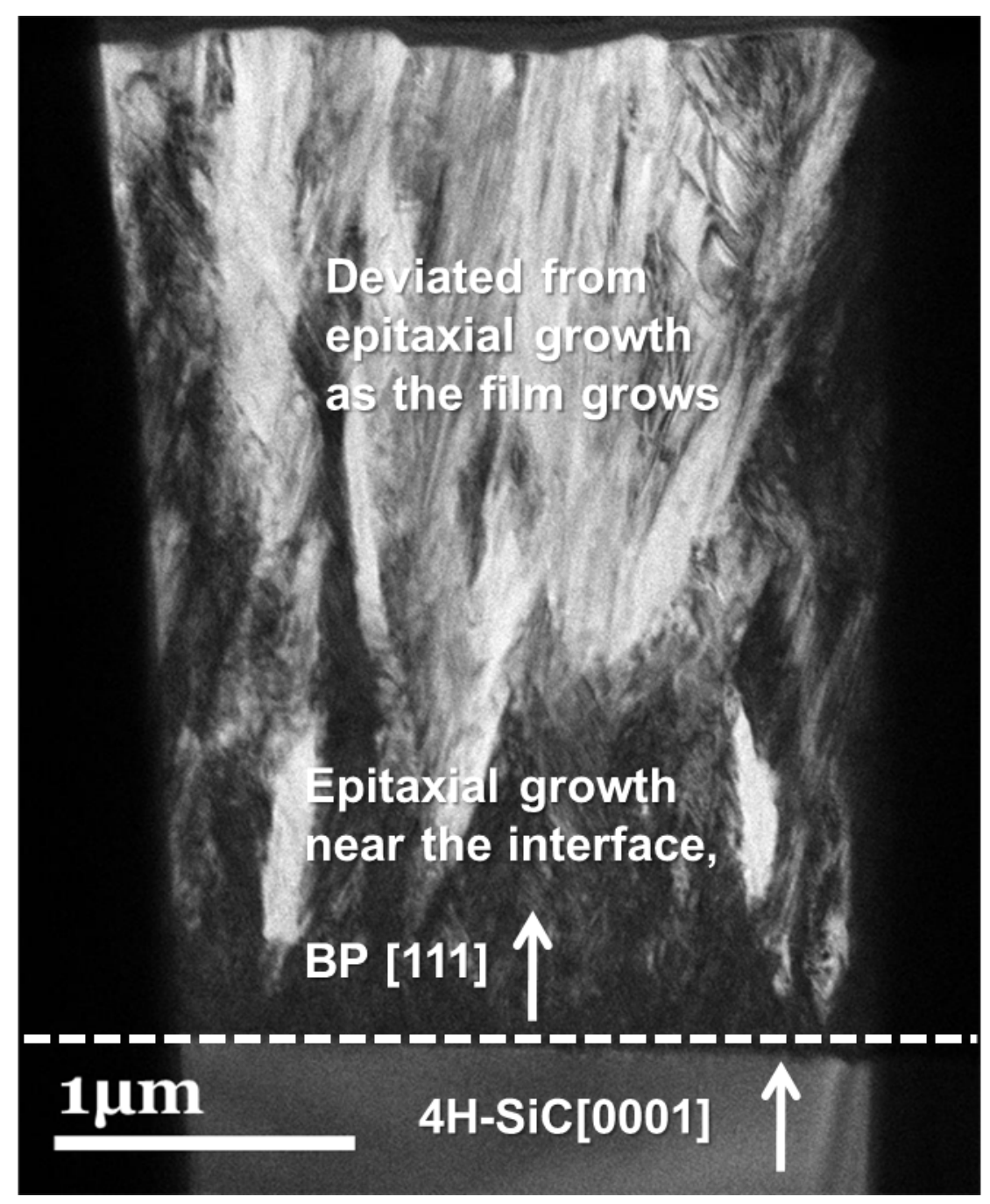

Fig. 1. An overview bright-field TEM image of the BP/SiC cross-section taken under a $\mathrm{SiC}<11-20>$ zoneaxis. The dark contrast near the interface indicate epitaxial growth, the bright contrast in the upper part indicates the epitaxial growth is not retained as the film develops.

To determine the structural evolution of the BP film, selected area diffraction (SAD) experiments were performed under a $<11-20>$ zone-axis of $\mathrm{SiC}$ throughout the entire film. The SAD aperture diameter was approximately $850 \mathrm{~nm}$. The results are shown in Fig. 2 where the approximate regions that contributed to the diffractions are indicated by a circle in the bright- 
field TEM image. While details of the diffraction pattern analysis will be discussed in the following text, the following sequence will serve as an overview:

(a). $4 \mathrm{H}-\mathrm{SiC}$ and $\mathrm{BP}$ interface: The overlap of $\mathrm{SiC}(0001)$ and $\mathrm{BP}$ (111) diffraction spots indicates that the epitaxial relationship of $\mathrm{BP}$ with $\mathrm{SiC}$ is $\mathrm{SiC}(0001),<11-20>/ /$ FCC BP (111), $<110>$.

(b). Near-interface region $(<1 \mu \mathrm{m})$ : The SAD pattern consists of (1) mutually-mirrored BP $<110>$ diffraction patterns with similar intensities, indicating the formation of a large amount of $\Sigma 3$ coherent twins boundaries (CTBs), (2) forbidden diffraction spots such as $1 / 3\{224\}, 1 / 3\{111\}$ and $2 / 3\{111\}$, indicating the existence of $\Sigma 3$ incoherent twin boundaries (ITBs), and (3) strong diffuse streaks along $<111>$ directions, indicating the presence of $\{111\}$ planar defects.

(c). Mid-film region $(\sim 1-2 \mu \mathrm{m})$ : One of the mutually-mirrored BP $<110>$ patterns becomes much brighter than the other. This means one BP crystal grew preferentially over its twinned counterpart. The TEM image reveals that the average grain-size in the $1 \sim 2 \mu \mathrm{m}$ region is greatly increased. The weaker diffuse streaks suggest less $\{111\}$ planar defects. The presence of the unknown diffraction spots are from the above-mentioned non-epitaxial crystals, which imply the emergence of randomly orientated grains.

(d). Near-surface region $(\sim 2-3 \mu \mathrm{m})$ : Grain boundaries are the main defects. 


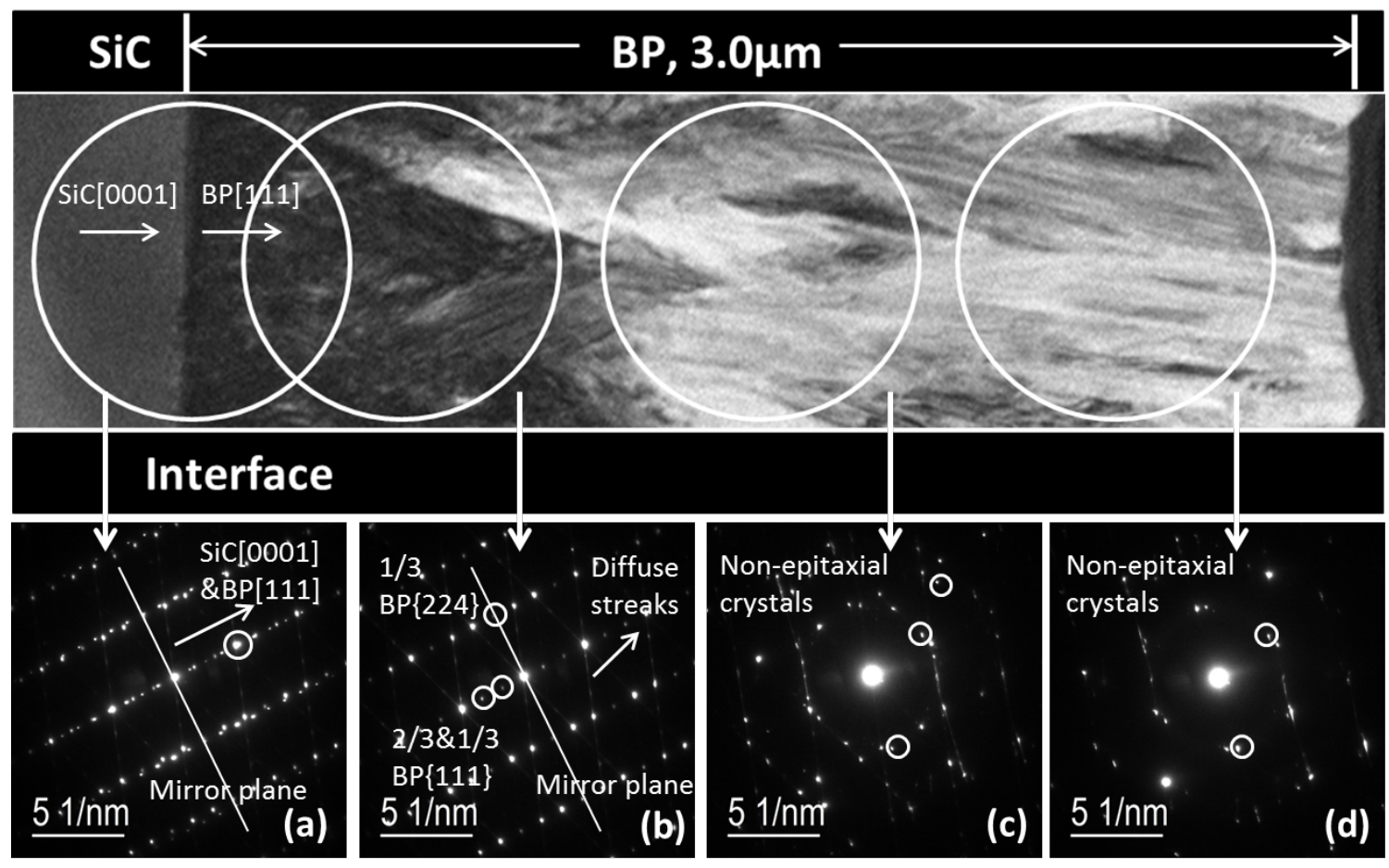

Fig. 2. Sequential $S A D$ patterns taken under a $S i C<11-20>$ zone-axis. (a) is the SAD pattern of the $4 H-$ $\mathrm{SiC} / \mathrm{BP}$ interface. The epitaxial relationship of BP with $\mathrm{SiC}$ is $\mathrm{SiC}(0001),<11-20>/ /$ FCC BP (111), <110>. (b) represents the-near interface region. The mirrored pattern and diffuse streaks imply twin boundaries and planar defects, respectively. (c) shows the mid-film region. Less twins and planar defects are observed, instead, grain boundaries between epitaxial and non-epitaxial BP crystals (circled diffraction spots) become the main defects. (d) is the surface region. Grain boundaries are the dominating defects.

\subsection{Detailed structure characterization}

The structural evolution of the BP film reflects the growth and strain relief mechanisms, therefore, is essential for future growth control and optimization. We will start from the $\mathrm{BP} / \mathrm{SiC}$ interface and characterize all the structures emerged along the BP growth direction.

\subsubsection{Misfit dislocations}


Aberration-corrected scanning transmission electron microscope (STEM) was used to study the atomic structures at the interface. The image below (Fig. 3) was acquired with the dedicated STEM VG HB603 at Oak Ridge National Laboratory. Counting the atoms, we found that 108 BP $<111>$ atomic columns correspond to $112 \mathrm{SiC}<0001>$ atomic columns. The statistics indicate that there is one misfit dislocation in every $28 \mathrm{SiC}$ lattice planes, or one misfit dislocation per $7.5 \mathrm{~nm}$ if viewed in a $\mathrm{SiC}<11-20>$ zone-axis. Assuming that the $\mathrm{SiC}$ lattice is not distorted and using it as a gauge, then the calculated BP $\{111\}$ d-spacing would be $3.16 \mathrm{~A}$ which results in a $1.6 \%$ misfit at this interface. The theoretical misfit of $\mathrm{BP}$ and $\mathrm{SiC}$ is, however, $4.5 \%$, which means that BP is still strained by the interface. This finding is consistent with the results of the strain analysis in the later discussion. The role of these misfit dislocations is to release the strain from $\mathrm{BP} / \mathrm{SiC}$ lattice mismatch. However, there are not enough of these dislocations present to fully release the strain and the residual strain seems to interact with the subsequently formed twins, dislocation loops and stacking faults. 


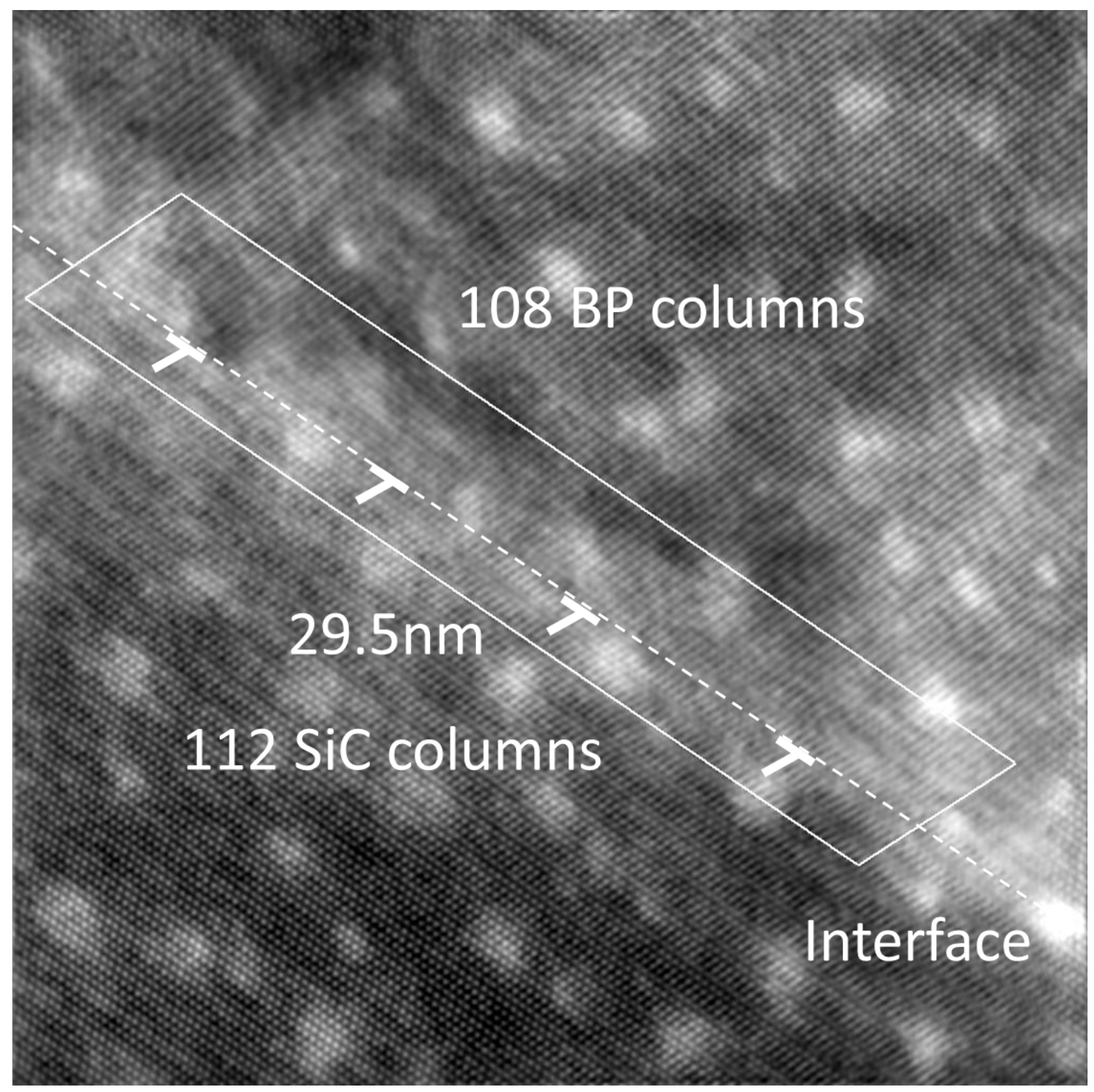

Fig. 3. Z-contrast image of the interface taken in a SiC $<11-20>$ zone-axis. $108 \mathrm{BP}<111>$ atomic columns correspond to $112 \mathrm{SiC}<0001>$ atomic columns, which is about $30 \mathrm{~nm}$ in distance. This means there are 4 misfit dislocations in every $30 \mathrm{~nm}$.

\subsubsection{Twin boundaries}

Based on the SAD analysis, twin boundaries are the most abundant defects in the near-interface region of BP. When stacking a $\mathrm{BP}$ (111) plane which has a 3-fold symmetry, on a 4H-SiC (0001) plane which has a six-fold symmetry, two sequences ("ABC" and "ACB") are available. The 
effect of the symmetric relationships between different stacking sequences on twin boundary formations has been theoretically treated by Grundmann [11]. $\Sigma 3$ CTBs are formed when ABCor ACB sequenced stacks pile up on each other, and exhibit complete coherency at the boundaries without straining of lattices; while $\Sigma 3$ ITBs are formed when $\mathrm{ABC}$ - and $\mathrm{ACB}$ sequenced stacks meet horizontally, which cause extended distortion. The $\Sigma$ 3CTBs and $\Sigma 3$ ITBs appear as stripes and pyramids, respectively, if viewed along a $\mathrm{BP}<110>$ zone-axis. The coexistence of the two types of twin boundaries is well demonstrated in the second SAD pattern in Fig. 2. As was mentioned above, the mutually mirrored $\mathrm{BP}<110>$ diffraction patterns are due to coherent twin boundaries. The $1 / 3\{224\}, 1 / 3\{111\}$ and $2 / 3\{111\}$ diffraction spots which are not supposed to exist in a FCC diffraction pattern are due to coincidence site lattice of the $\Sigma 3$ ITBs (Fig. 3(b)), or to be specific, their formation is due to the changes of periodicities in both $<224>$ and $<111>$ directions. Note, these twin boundaries serve as either a source or a sink of the strain and would interact with the residual strain which was not released by the misfit dislocations.

Fig. 4 clearly shows the origin and characterization of the $\Sigma 3$ ITBs, which are similar to the double positioning boundaries formed in epitaxial 3C-SiC grown on $6 \mathrm{H}-\mathrm{SiC}$ [12]. The diffractogram generated at the $\Sigma 3$ ITBs contains the features of both diffractograms of BP grown on the two sides. The SEM image embedded in the HRTEM image shows the coalescence of two twinned BP nuclei. The twinning relationship was inferred from the opposite facet-orientations of the top surface. The atomic model schematically describes the strain distribution near the $\Sigma 3$ ITBs. 


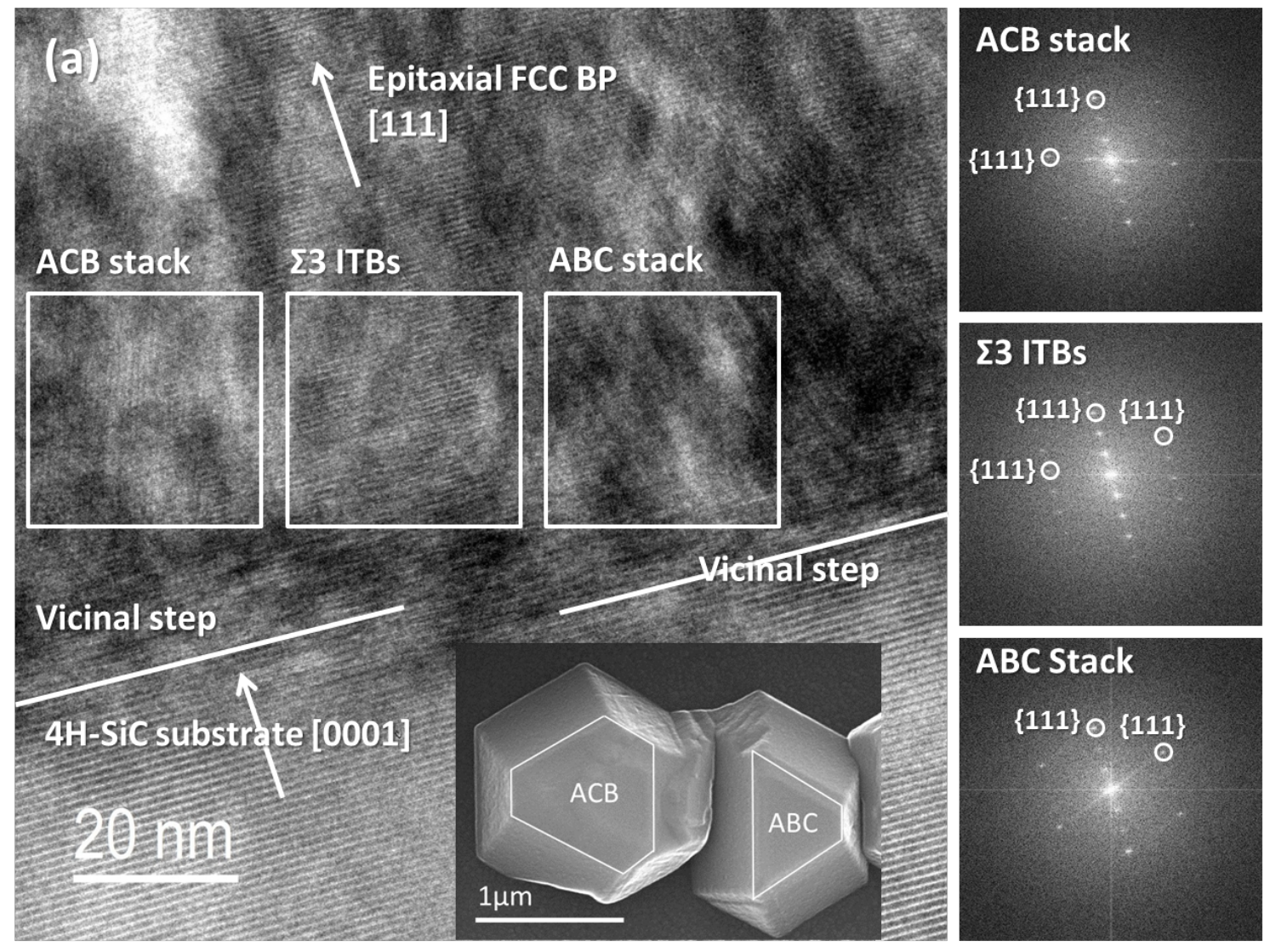

(b)

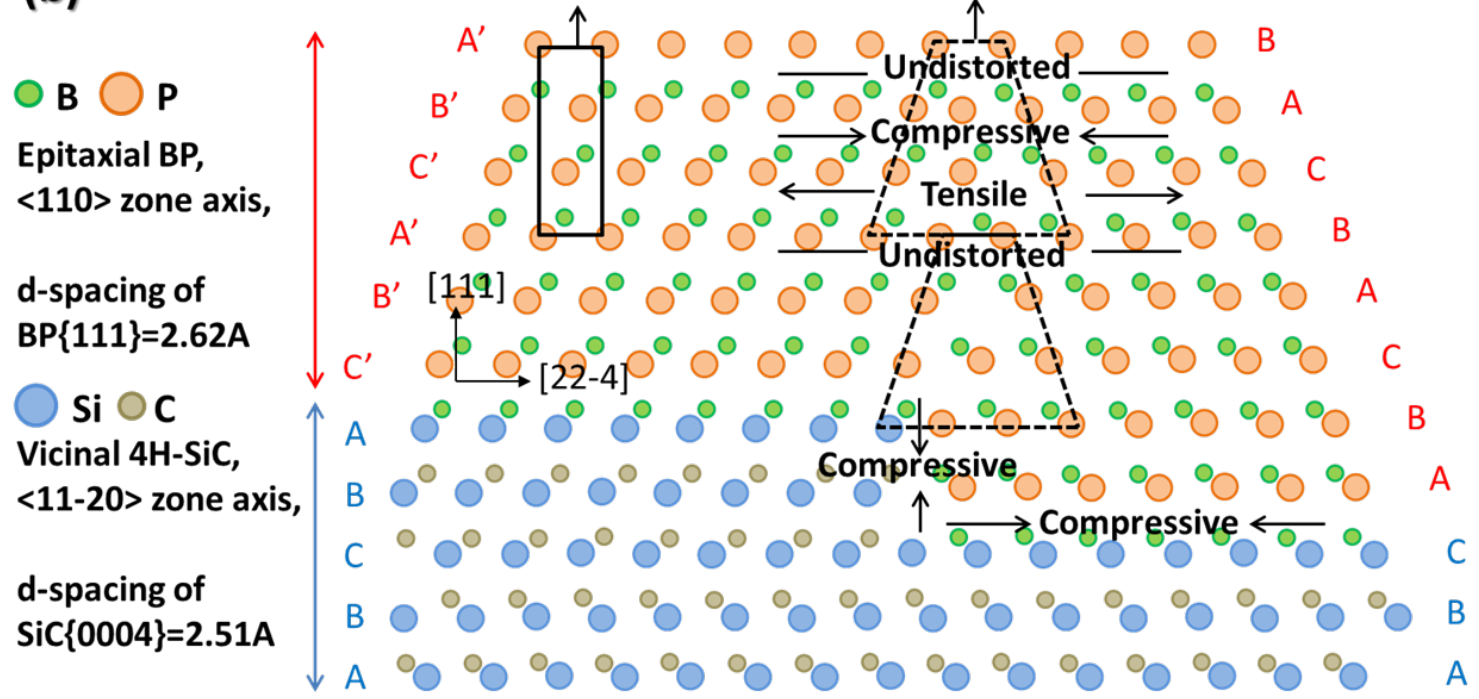

Fig. 4. Origin and characterization of $\Sigma 3$ ITBs. (a) The HRTEM image shows the formation of $\Sigma 3$ ITBs on the vicinal SiC substrate. The embedded SEM image shows the coalescence of two twinned BP nuclei. The diffractogram of the $\Sigma 3$ ITB contains the features of both diffractograms of BP grown on the 
neighboring surfaces. (b) is the atomic model of the microstructure at the vicinal step. $\Sigma 3$ ITBs, coincidence site lattice, strain types are labeled. (Misfit dislocations are not shown).

\subsubsection{Dislocation loops and stacking faults}

If, for example, the additional lattice plane is not a half-plane but of finite size, a dislocation loop is formed at the boundary of the dislocation lines. If, the stacking-sequence of the crystal structure is interrupted by one or two atomic layers, then a stacking fault is generated. Dislocation loops and stacking faults are widely distributed planar defects in our BP films. Their dimensions are limited by the twin boundaries in the near-interface region and increase when this limitation is reduced further away from the interface. Fig. 5(a) is a HRTEM image illustrating the two types of defects. The stripes circled in Fig. 5 represent dislocation loops or stacking faults which all lie on the $\{111\}$ close packed planes. The contrast of the stripes are caused by the diffraction contrast of the defect planes. The pattern inside is the diffractogram of the HRTEM image, and it consists of a typical FCC BP $<110>$ diffraction pattern and intense diffuse streaks. The diffuse streaks result from the Fourier transform of $\{111\}$ planar defects. Analysis of the diffuse streaks provides information on the orientations, dimensions and densities of the planar defects. Fig. 5(b) and (c) are Z-contrast images showing a dislocation loop and a stacking fault, respectively. 

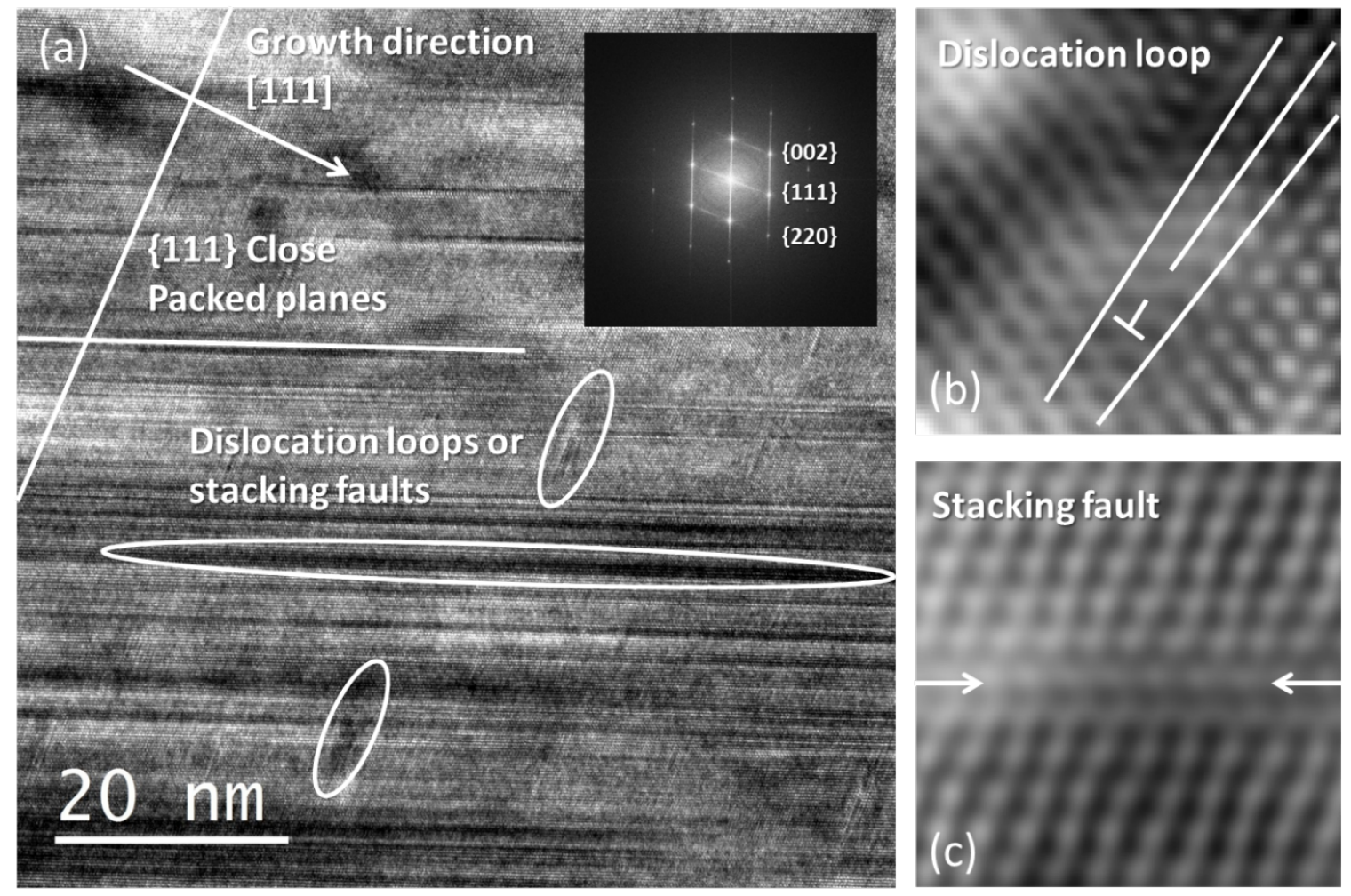

Fig. 5. (a). HRTEM image of the planar defects in BP; the pattern inside is the corresponding diffractogram. The diffuse streaks in the diffractogram are caused by the planar defects. The planar defects are circled in the TEM image. (b) Z-contrast image of one end of a dislocation loop. (c) Z-contrast image of a stacking fault.

\subsubsection{Grain boundaries}

Grain boundaries are the main defects further away from the interface. In the near-interface region, BP crystals exhibit pyramidal morphology which suggests epitaxial growth. However, non-epitaxial columnar growth emerges as the film develops, and their formations are due to the coalescence of the nuclei or further grown crystals. The growth orientations of the columnar crystals are unpredictable. In a previous study of BP growth on (100) Si, where crystals nucleated from an amorphous base, the preferred growth direction of BP was $<110>$. Development of non-epitaxial columns of BP may be driven by this preferential growth direction. 
The growth mechanism is best illustrated in Fig. 6, which mixes the dark field images taken with different diffraction beams. The distribution of the epitaxial and non-epitaxial BP crystal is mapped. The red area corresponds to epitaxial growth. All the other colored areas represent nonepitaxial columnar growths with different orientations. From the image, we know that epitaxial growth starts at the interface and competes with the non-epitaxial growth which originated at the pits of the epitaxial pyramids.
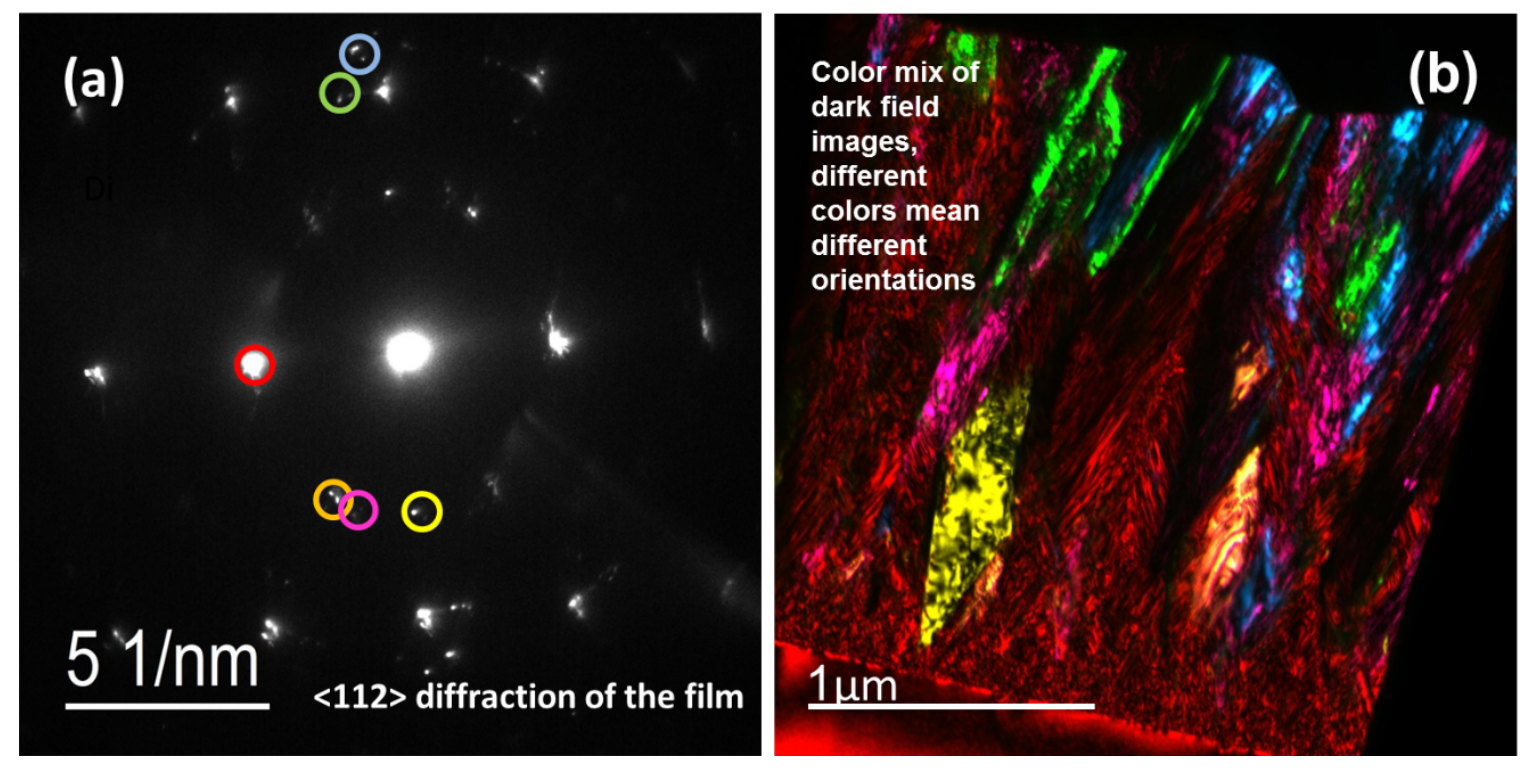

Fig. 6. Distribution of epitaxial and non-epitaxial BP crystals within the film. (a) is the diffraction pattern of the BP film taken under $a<112>$ zone-axis. The cirlced diffraction spots are colored to correspond with the stacked dark-field images in Fig. b. (b). Epitaxial growth starts at the interface and competes with the non-epitaxial growth originating at the pits of the epitaxial pyramids.

\subsection{Strain relief analysis}

Lattice distortions of the near-interface region of the BP film were investigated using diffractograms of the HRTEM images. Thirteen HRTEM images were taken starting from the interface with step size of approximately 100nm. The d-spacings were calculated by applying 
Bragg's law to the corresponding diffractograms and compiled in a plot (Fig. 7). This method yields very minimum error. It is worth noting that lattice distortions are measured using discontinuous HRTEM images, therefore, the distortion evolution does not necessarily follow the lines between the data points. The d-spacing evolution in the growth direction is closely related to the density of twin boundaries. According to the above structural analysis, the first eight HRTEM images were located in the near-interface region where twin boundaries were concentrated, while the subsequent five HRTEM images were away from the twin-dominated region. Therefore, the eighth data point differentiates where the BP crystal lattice contracted and then expanded along the growth direction. However, the d-spacings in the other $\{111\}$ close packed plane were under contraction throughout the examined region. Fig. 7 suggests that the formation of misfit dislocations and subsequent defects did not fully release the strain originating from the $4.5 \%$ lattice mismatch. This is a general observation in all our epitaxial BP samples. We conclude that the complex strain-field in the near-interface region prohibited the formation of threading dislocations which normally propagates throughout the whole epitaxial film [13]. The formation of defects throughout the film are all considered to play the role of releasing strains, but they did not eliminate the lattice distortion. These defects can be used as engineering tools to modify the resistance and charge carrier mobilities to optimize the device performance. Further work is needed to correlate electrical and detector performance with specific defects in these materials. 


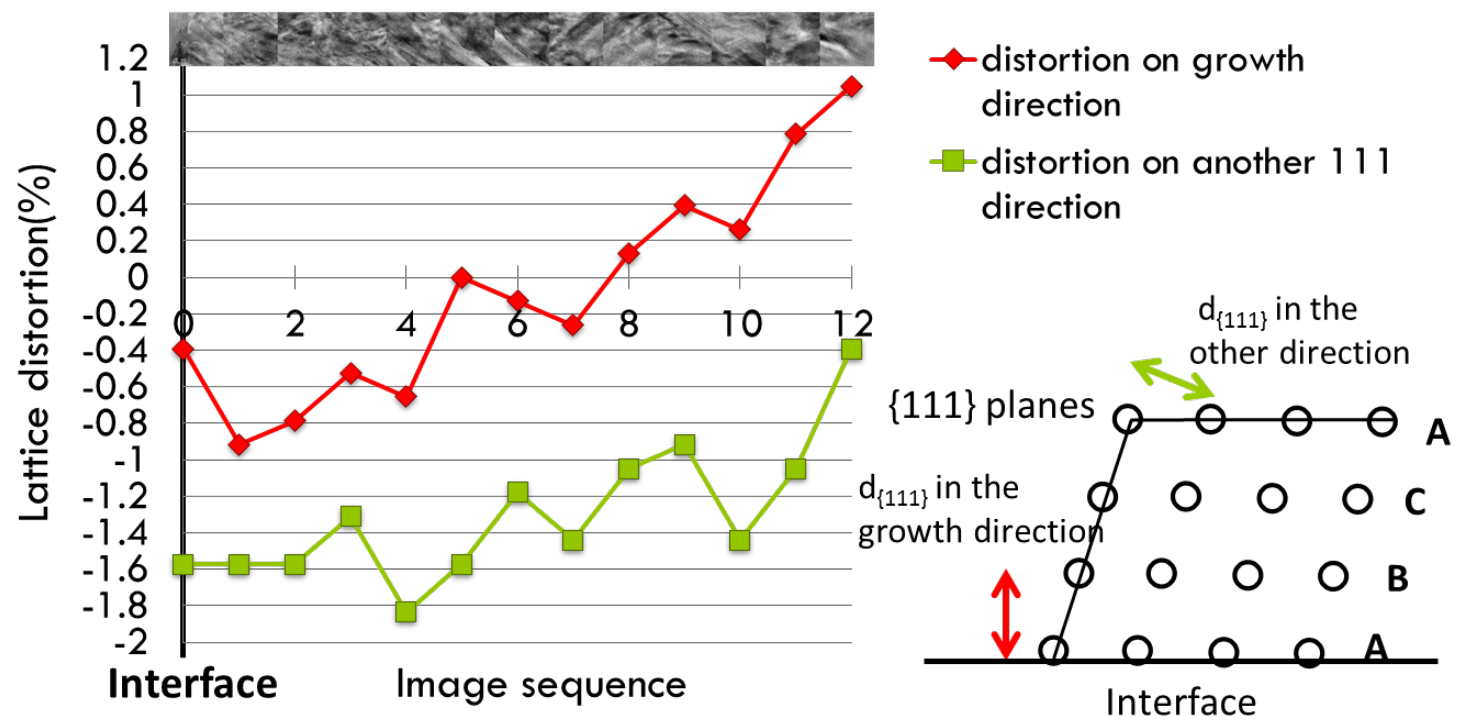

Fig. 7. The evolution of the lattice distortion. The red curve represents distortion along the [111] growth direction; the green curve represents distortion in the other $\{111\}$ close packed plane. The errors are smaller than the point markers in both cases, and the lines are included to guide the readers' eyes.

\section{Conclusions}

We conclude that the BP film growth on (0001) surfaces of $4 \mathrm{H}-\mathrm{SiC}$ substrates with a $4^{\circ}$ offcut begins as heteroepitaxial and develops into polycrystalline BP with a variation of defect densities and types. The BP film epitaxially grew at the interface with two different stacking sequences. The near-interface region is densely occupied by small domains segmented by twin boundaries, stacking faults and dislocation loops. These defects played the role of releasing the strain originated from the $4.5 \%$ lattice mismatch, however, they did not eliminate the strain. As the film develops, large-sized grains started to emerge and grain boundaries became the dominating defects in the film. We project that further improvement in film quality should be possible through rapid feedback between TEM investigations and synthesis. 


\section{Acknowledgements}

The research is supported by the U.S. Department of Energy (DOE), NNSA, NA-221, Office of Nuclear Proliferation Detection. We thank Joint Institute for Advanced Materials (JIAM) at the University of Tennessee and DOE-Basic Energy Sciences (BES) for microscope access.

\section{References}

[1] G.F. Knoll, Radiation Detection and Measurement, John Wiley \& Sons, 2010.

[2] T. Nishinaga, H. Ogawa, H. Watanabe, T. Arizumi, Vapor growth of boron monophosphide using open and closed tube processes, Journal of Crystal Growth, 13-14 (1972) 346-349.

[3] K. Shohno, M. Takigawa, T. Nakada, Epitaxial growth of BP compounds on Si substrates using the B2H6-PH3-H2 system, Journal of Crystal Growth, 24-25 (1974) 193-196.

[4] M. Iwami, T. Tohda, K. Kawabe, Crystal growth of boron mono-phosphide and its electrical and optical properties, Electrical Engineering in Japan, 95 (1975) 19-24.

[5] Y. Kumashiro, Y. Okada, S. Gonda, Crystal growth of thick wafers of boron phosphide, Journal of Crystal Growth, 70 (1984) 507-514.

[6] T. Yamashita, K. Yamatake, M. Odawara, T. Udagawa, Crystallographic characterization of hetero-epitaxial growth manner of BP semiconductor on (111)-Si, Thin Solid Films, 464-465 (2004) 120-122.

[7] Y. Kumashiro, M. Matsumoto, H. Yoshizawa, Heteroepitaxial Growth of Boron Phosphide Single Crystal on Sapphire Single Crystal, JJAP series, 10 (1994) 160-163.

[8] Y. Kumashiro, K. Nakamura, T. Enomoto, M. Tanaka, Preparation and thermoelectric properties of BP films on SOI and sapphire substrates, J Mater Sci: Mater Electron, 22 (2011) 966-973.

[9] T.L. Chu, J.M. Jackson, A.E. Hyslop, S.C. Chu, Crystals and Epitaxial Layers of Boron Phosphide, Journal of Applied Physics, 42 (1971) 420-424.

[10] T. Udagawa, M. Odawara, G. Shimaoka, High-resolution TEM characterization of MOVPE-grown (111)-BP layer on hexagonal 6H (0001)-SiC, Applied Surface Science, 244 (2005) 285-288.

[11] M. Grundmann, Formation of epitaxial domains: Unified theory and survey of experimental results, physica status solidi (b), 248 (2011) 805-824.

[12] H.S. Kong, B.L. Jiang, J.T. Glass, G.A. Rozgonyi, K.L. More, An examination of double positioning boundaries and interface misfit in beta-SiC films on alpha-SiC substrates, Journal of Applied Physics, 63 (1988) 2645-2650.

[13] J.P. Douglas, $\mathrm{Si} / \mathrm{SiGe}$ heterostructures: from material and physics to devices and circuits, Semiconductor Science and Technology, 19 (2004) R75. 